\title{
Restorative effects of hydroxysafflor yellow A on hepatic function in an experimental regression model of hepatic fibrosis induced by carbon tetrachloride
}

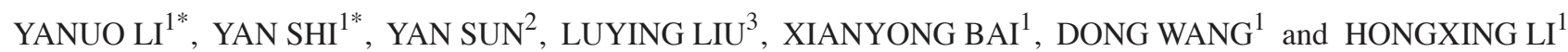 \\ ${ }^{1}$ Department of Histology and Embryology, Binzhou Medical University, Yantai, Shandong 264003; \\ ${ }^{2}$ Department of Osteology, Yantaishan Hospital, Yantai, Shandong 264000; ${ }^{3}$ Department of Pathology, \\ Binzhou Medical University, Yantai, Shandong 264003, P.R. China
}

Received September 18, 2015; Accepted September 16, 2016

DOI: $10.3892 / \mathrm{mmr} .2016 .5965$

\begin{abstract}
Hepatic fibrosis is a reversible pathological process, in which fibrotic tissue is excessively deposited in the liver during the repair process that follows hepatic injury. Early prevention or treatment of hepatic fibrosis has great significance on the treatment of chronic hepatic diseases. Hydroxysafflor yellow A (HSYA) is a water-soluble monomer extracted from safflower, which serves numerous pharmacological roles. However, it remains to be elucidated how HSYA regulates hepatic fibrogenesis. The aim of the present study was to reveal the possible mechanisms underlying the effects of HSYA on the prevention and treatment of hepatic fibrosis. A rat model of hepatic fibrosis was established in the present study, and the rats were administered various doses of HSYA. The effects of HSYA on pathological alterations of the liver tissue in rats with hepatic fibrosis were observed using hematoxylin-eosin staining and Masson staining. In order to explore the anti-hepatic fibrosis effects and underlying mechanisms of HSYA, serum levels, and hepatic function and hepatic fibrosis indices were evaluated. The results demonstrated that HSYA can improve the general condition of rats with hepatic fibrosis and relieve cellular swelling of the liver, fatty degeneration, necrosis, inflammatory cell infiltration and fibroplastic proliferation. Subsequent to administration of HSYA, globulin was increased during hepatic fibrosis caused by tetrachloromethane. However, total cholesterol, triglyceride, alanine aminotransferase, aspartate aminotransferase and levels of hyaluronic acid, laminin, procollagen III N-terminal peptide, collagen
\end{abstract}

Correspondence to: Professor Yanuo Li, Department of Histology and Embryology, Binzhou Medical University, 346 Guanhai Road, Laishan, Yantai, Shandong 264003, P.R. China

E-mail: yaya-698@163.com

*Contributed equally

Key words: hydroxysafflor yellow A, hepatic fibrosis, carbon tetrachloride, hepatic function type IV and hydroxyproline were significantly reduced. The results additionally demonstrated that HSYA could enhance superoxide dismutase activity and reduce malondialdehyde levels, inhibiting lipid peroxidation caused by free radicals.

\section{Introduction}

Hepatic fibrosis is a common process that occurs in the liver in response to diverse chronic injury. Factors associated with hepatic injury can result in hepatic fibrosis, including viral hepatitis, steatohepatitis (including alcoholic and nonalcoholic), autoimmune hepatic disease, Schistosomiasis japonica infection, toxic or drug-induced hepatic disease, and genetic and metabolic diseases (1). During the pathological process of hepatic fibrosis the following symptoms are observed: Decreased normal liver cells, diffuse liver cell necrosis, abnormal proliferation of connective tissue, and disordered hepatic circulation, which can lead to hepatic function failure and subsequently to hepatic cirrhosis (2). A previous study reported that hepatic fibrosis is a reversible pathological phenomenon, in which fibrotic tissue is excessively deposited in the liver during the repair process that follows hepatic injury. Early prevention or treatment of hepatic fibrosis has great significance on the treatment of chronic hepatic diseases (3). Although there have been some drugs used to treat this pathogenic process, the efficacy fails to meet necessary requirements. At present, researchers aim to seek drugs with definite efficacy and few adverse effects in order to delay, suppress or even reverse the process of hepatic fibrosis. Several clinical practices and experimental studies have demonstrated that traditional Chinese medicine can relieve hepatic fibrosis and hepatic cirrhosis via multi-component, multi-path, multi-level and multi-target comprehensive pharmacological effects (4-6). In order to further investigate the antifibrotic action of traditional Chinese medicines, several scholars have aimed to research drugs extracted from medicinal plants and to explore the underlying antifibrotic mechanisms of action.

Safflower (Carthamus tinctorius L.), which belongs to the Compositae family, consists of tubular florets and is distributed in the Mediterranean and Asia. Safflower is a traditional drug used for activating circulation, dissolving 
stasis, activating collaterals and alleviating pain (7). The main components of safflower include pigment, flavonoids, volatile oils, fatty acids and phenolic acids (8). The yellow pigment of safflower is predominantly used for activating blood and dissolving stasis. Hydroxysafflor yellow A (HSYA) is a water-soluble monomer that is extracted from safflower and is a type of chalcone glycoside (molecular formula, $\mathrm{C}_{27} \mathrm{H}_{32} \mathrm{O}_{16}$; molecular weight, $612.53 \mathrm{~g} / \mathrm{mol}$ ) (9). Previous studies have demonstrated that HSYA possesses several functions, including widening of coronary arteries and ameliorating myocardial ischemia, anticoagulation, anti-platelet aggregation and relief from unstable angina pectoris, protecting against brain and spinal cord ischemia-reperfusion injury, and preventing steroid-induced avascular necrosis of the femoral head $(10,11)$. In addition, it has been reported that HSYA is able to eliminate free radicals, inhibit lipid peroxidation, relieve inflammatory injury and suppress cell apoptosis (12). HSYA has been demonstrated to reduce pulmonary fibrosis (13); however, few studies have investigated the role of HSYA in hepatic fibrosis.

The present study was performed using a rat model of hepatic fibrosis induced by carbon tetrachloride $\left(\mathrm{CCl}_{4}\right) \cdot \mathrm{CCl}_{4}$ is often used to generate a reliable animal model of hepatic fibrosis (14). This model simulates the clinical pathological process of hepatic fibrosis, and provides an ideal animal model for analyzing the anti-hepatic fibrosis effect of drugs. $\mathrm{CCl}_{4}$ is a selective liver toxicant that can induce liver cell necrosis. Treatment with low-dose $\mathrm{CCl}_{4}$ for a long duration can induce hepatic fibrosis in animals. In addition, the degree of hepatic fibrosis is positively associated with the dose. Using the method of intragastric administration can reduce the mortality of animals. $\mathrm{CCl}_{4}$, together with ethanol and a high-fat diet, can accelerate liver cell injury, and trigger inflammation and fibrosis. The rat model of hepatic fibrosis was induced by $\mathrm{CCl}_{4}$, alongside administration of alcohol and a high-fat diet, which may accelerate liver cell injury, inflammation and fibrosis. With the use of the complex model combining carbon tetrachloride oxidative damage, alcoholism and lipid oxidative damage with a variety of factors, the formation mechanism of hepatic fibrosis is closer to that of clinical patients and is conducive to screening antifibrotic drugs.

The present study aimed to further explore the mechanism underlying the effects of HSYA on the prevention and treatment of hepatic fibrosis. In the present study, a rat model of hepatic fibrosis was established, and the rats were administered various doses of HSYA. The effects of HSYA on the pathological alterations of liver tissue in rats with hepatic fibrosis were observed using hematoxylin-eosin staining and Masson staining. In order to explore the anti-hepatic fibrosis effects and underlying mechanisms of HSYA, serum levels, and hepatic function and hepatic fibrosis indices were measured.

\section{Materials and methods}

Reagents. HSYA (purity $>98 \%$ ) was extracted from the herb Carthamus tinctorius L.; the water-soluble yellow amorphous powder was provided by Dr. Fenghua Fu (School of Pharmaceutical Sciences, Yantai University, Yantai, China). $\mathrm{CCl}_{4}$ (purity 99\%) was purchased from Tianjin Bodi Chemical Co., Ltd. (Tianjin, China). Colchicine was purchased from
Xishuangbanna Pharmaceutical Co., Ltd. (Xishuangbanna, China). Total protein (TP) quantitative assay kit (A045-2), albumin (ALB) assay kit (A028-1), total cholesterol (TCHO) assay kit (A111-1), triglyceride (TG) assay kit (A110-1), high-density lipoprotein cholesterol (HDL) assay kit (A112-1), low-density lipoprotein cholesterol (LDL) assay kit (A113-1), alanine aminotransferase (ALT) assay kit (C009-1), aspartate aminotransferase (AST) assay kit (C010-1), alkaline phosphatase (ALP) assay kit (C059-1), hydroxyproline (HYP) assay kit (A030-2), malondialdehyde (MDA) assay kit (A003-1) and superoxide dismutase (SOD) assay kit (A001-1) were obtained from Nanjing Jiancheng Bioengineering Institute (Nanjing, China). Hyaluronic acid (HA) radioimmunoassay kit (H141), laminin (LN) radioimmunoassay kit (H148), procollagen III $\mathrm{N}$-terminal peptide (pcIII) radioimmunoassay kit (H212) and collagen type IV (cIV) radioimmunoassay kit (H145) were purchased from Shanghai Haiyan Pharmaceutical Technology Co., Ltd. (Shanghai, China). Trichrome stain (Masson) kit was purchased from Fujian Maixin Biotechnology Development Co., Ltd. (Fuzhou, China).

Animals and group design. The current study was approved by the ethics committee of Binzhou Medical University (Yantai, China). The present study was conducted on male Sprague Dawley rats (age, 5-6 weeks; weight, 110-130 g), which were provided by the Experimental Animal Center of Shandong University [animal permit number: SUXK (Lu) 20130009; Jinan, China]. The rats were allowed to acclimate for 1 week, after which the rats that exhibited a normal reaction to food, water and activity, and no adverse effects, were enrolled in the study. Rats were maintained in a temperature-controlled room with an alternating 12-h dark and light cycle. A total of 10 rats were randomly selected as the normal control group (Group A). The remaining rats were randomly divided into six groups: Hepatic fibrosis model group (Group B), HSYA prevention group (Group C), low dose HSYA group (Group D), moderate dose HSYA group (Group E), high dose HSYA group (Group F) and the colchicine group (Group G). Each group consisted of 15 rats.

Animal model preparation. The rat hepatic fibrosis model was established using the $\mathrm{CCl}_{4}$ model method. Briefly, $\mathrm{CCl}_{4}$ and soybean oil were mixed at a ratio of $3: 7$, in order to obtain a $300 \mathrm{ml} / 1$ oil solution, which was administered by gavage at a dose of $3 \mathrm{ml} / \mathrm{kg}$, three times a week. From the $2 \mathrm{nd}$ week, $10 \%$ ethanol was added to the drinking water and $20 \%$ lard oil was added to the diet. Rats in Group A were administered $3 \mathrm{ml} / \mathrm{kg}$ soybean oil by gavage, three times a week, and were given ad libitum access to normal food and water.

Drug treatment. From the first week, rats in Groups B and C were given drug treatment for 10 weeks via intraperitoneal injection, five times a week. From the third week, rats in Groups D-G were given drug treatment for 8 weeks via intraperitoneal injection, five times a week. The treatments administered were as follows: Group B, injection of $1 \mathrm{ml} / \mathrm{kg}$ normal saline; Group C and E, injection of $10 \mathrm{mg} / \mathrm{kg}$ HSYA water solution; Group D, injection of $5 \mathrm{mg} / \mathrm{kg}$ HSYA water solution; Group F, injection of $20 \mathrm{mg} / \mathrm{kg}$ HSYA water solution; Group $\mathrm{G}$, injection of $0.1 \mathrm{mg} / \mathrm{kg}$ colchicine. HSYA and 
colchicine were dissolved in normal saline for filtration and sterilization.

Sample collection and detection. Following establishment of the rat model, the growth conditions of the rats in each group were observed; the rats were weighed and a weight growth curve was generated. At the $10^{\text {th }}$ week, all rats were fasted for $24 \mathrm{~h}$ and were then administered $3.5 \%$ chloral hydrate. After blood samples were collected via heart puncture, rats were sacrificed via excessive blood loss. Blood samples were collected via heart puncture, were maintained at room temperature for $1 \mathrm{~h}$, and were centrifuged at 3,000 r/min for $15 \mathrm{~min}$ at $4^{\circ} \mathrm{C}$ to separate serum, serum levels (TP, ALB, TCHO, TG, HDL, LDL, ALT, AST and ALP) were detected according to manufacturer's protocols. GLB content is equal to the total protein content minus the albumin content (15). The livers were also collected, separated and weighed to calculate the liver to body weight ratio. Furthermore, tissue samples were collected from the left lobe of the liver, fixed with paraformaldehyde, embedded with paraffin and cut into $4 \mu \mathrm{m}$ sections. The sections underwent hematoxylin-eosin (hematoxylin for $10 \mathrm{~min}$ and eosin for $3-5 \mathrm{~min}$ both at $25^{\circ} \mathrm{C}$ ) and Masson $(5 \mathrm{~min}$ at $25^{\circ} \mathrm{C}$ ) staining, and were observed under a microscope (DM4000B; Leica Microsystems GmbH; Wetzlar, Gernman). Tissue samples collected from the right lobe of the liver were weighed, homogenized and detected according to manufacturer's protocols.

Statistical analysis. Statistical analysis was performed using software SPSS 17.0 (SPSS, Inc., Chicago, IL, USA). All data in the present study are expressed as $\mathrm{x} \pm \mathrm{s}$. Data were analyzed using homogeneity of variances test and intergroup variance analysis. $\mathrm{P}<0.05$ was considered to indicate a statistically significant difference.

\section{Results}

Effects of HSYA on physiological state, weight and liver/body ratio in rats with hepatic fibrosis. A total of 20 rats died during the present study (one rat in Group A, four in Group B, three in Group C, four in Group D, three in Group E, three in Group F and two in Group G). Rats in the normal group were lively and exhibited a good appetite, with shiny fur and increased weight. After the model was established, rats exhibited a decreased appetite, little activity, low spirits, and had dry and lackluster fur. From the third week, rat weight was significantly decreased in Group B compared with Group A ( $\mathrm{P}<0.01$; Fig. 1A). Following administration of HSYA and colchicine, food intake, mood and fur condition were improved compared with in the rats in Group B. As the duration of HYSA and colchicine administration increased, the weight of the rats increased compared with in the model group. After 9 weeks, rat weight in Groups C, E and $\mathrm{F}$ was higher than in Group $\mathrm{B}(\mathrm{P}<0.01)$. Furthermore, the liver tissue of the normal rats had a smooth surface, bright reddish color and was soft to the touch. The liver tissue of rats in Group B had a rough surface, increased volume, pale color, was hard to the touch, and fine particles were observed on the surface. In addition, some rats exhibited hepatic portal vein varices, and the rats that died exhibited fluid retention in the abdominal cavity. Compared with Group A, the liver/body ratio in Group B was increased ( $\mathrm{P}<0.01$; Fig. 1B). Liver morphology and color were slightly ameliorated in the drug-treated rats compared with in Group B; however, fine particles were still observed. Compared with Group B, liver/body weight ratio was decreased, particularly in Groups $\mathrm{C}$ and $\mathrm{E}$ ( $\mathrm{P}<0.01$; Fig. 1B).

Effects of HSYA on pathological alterations of liver tissue in rats with hepatic fibrosis. Under a light microscope, hematoxylin-eosin staining demonstrated that hepatic lobules in Group A rats were clear with an intact structure, the central vein was surrounded by cords of liver cells that radiated out in all directions, irregular hepatic sinusoid was seen among the hepatic cords, and liver cells exhibited no necrosis or inflammatory cell infiltration (Fig. 2A). Parenchymal nodules, varying from small to large, which are encircled by fibrotic bands were observed, and these nodules commonly contain proliferating hepatocytes (Fig. 2B). Compared with Group B, hepatic lobules in drug-treated rats (Groups $\mathrm{C}-\mathrm{G}$ ) exhibited reduced structural damage, decreased pseudolobules, cellular swelling, fatty degeneration, necrosis and inflammatory cell infiltration, particularly in Group C (Fig. 2C). Masson staining results demonstrated that the liver calls of rats in Group A were arranged normally and few blue collagen fibers could be seen in the portal area (Fig. 2H). Conversely, liver tissue of rats in Group B exhibited marked collagen fiber proliferation, predominantly in portal areas and the central vein, and pseudolobule formation was detected (Fig. 2I). Liver tissue of rats in the drug-treated group (Groups C-G) exhibited decreased deposition of collagen fibers, thin fibrous septum and decreased pseudolobule formation (Fig. 2J)

Effects of HSYA on protein metabolism in rats with hepatic fibrosis. Compared with Group A, serum TP and ALB levels in Group B were significantly decreased $(\mathrm{P}<0.01$; Figs. 3A and B); globulin (GLB) was increased $(\mathrm{P}<0.01$; Fig. 3C) and the albumin/globulin ratio (A/G) was decreased $(\mathrm{P}<0.01$; Fig. 3D). As compared with Group B, HYSA and colchicine treatment resulted in an increase in ALB and $\mathrm{A} / \mathrm{G}$ ratio. In particular, serum ALB levels were increased in Groups $\mathrm{C}$ and $\mathrm{E}(\mathrm{P}<0.01)$.

Effects of HSYA on lipid metabolism in rats with hepatic fibrosis. Compared with Group A, serum TCHO, TG, HDL and LDL levels were significantly increased in Group B ( $\mathrm{P}<0.01$; Fig. 4). As compared with Group B, following HSYA and colchicine treatment, TCHO and TG were significantly decreased $(\mathrm{P}<0.05)$, and HDL and LDL had the tendency to decrease; however, no significant differences were detected in HDL levels $(\mathrm{P}>0.05)$.

Effects of HSYA on serum ALT, AST and ALP levels in rats with hepatic fibrosis. Compared with Group A, serum ALT, AST and ALP levels in Group B were significantly increased $(\mathrm{P}<0.01)$ and the DeRitis value $(\mathrm{AST} / \mathrm{ALT})$ was decreased $(\mathrm{P}<0.01$; Fig. 5). Compared with Group B, following treatment with HSYA and colchicine, serum ALT and ALP levels were decreased, particularly in Groups C, E and G $(\mathrm{P}<0.05)$. Serum AST had the tendency to decrease; however, no significant differences were detected $(\mathrm{P}>0.05)$. In addition, the DeRitis value was significantly increased in Groups $\mathrm{C}$ and $\mathrm{E}(\mathrm{P}<0.05)$. 
A

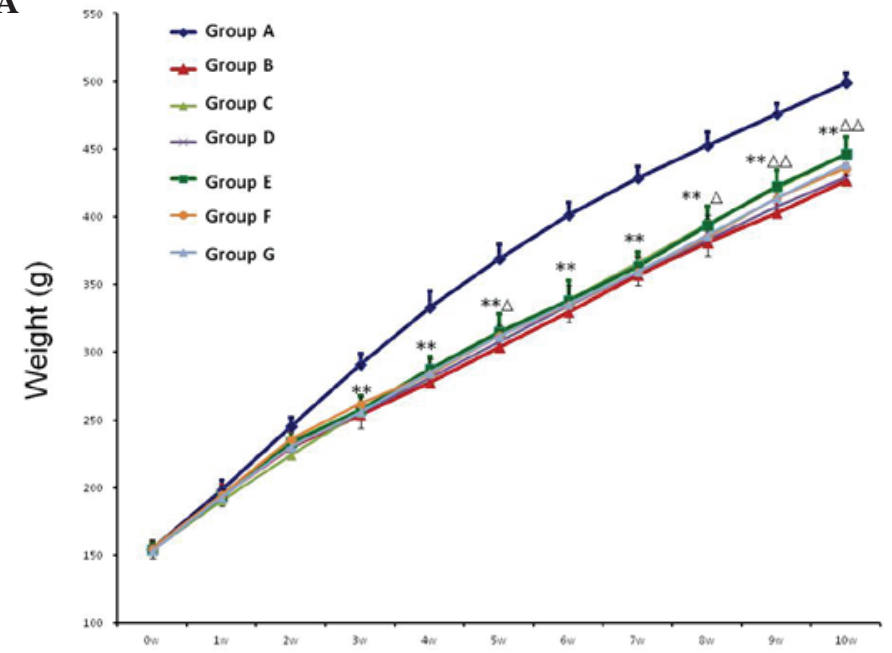

B

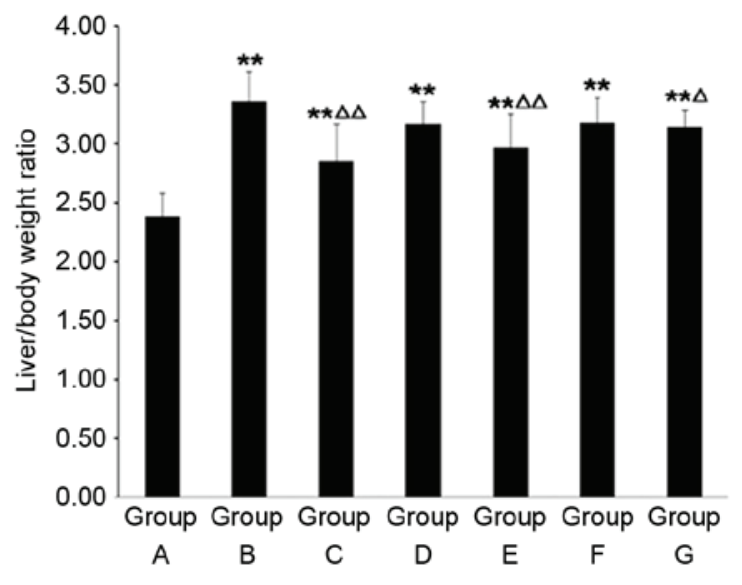

Figure 1. Effects of HSYA on (A) body weight and (B) liver/body weight ratio of rats. From the second week, rats in the model group exhibited naturally decreased body weight, and liver/body weight ratio was increased compared with in Group A. ${ }^{* *} \mathrm{P}<0.01$ vs. Group A; ${ }^{\Delta} \mathrm{P}<0.05,{ }^{\Delta \Delta} \mathrm{P}<0.01$ vs. Group B. Group A, normal control; Group B, hepatic fibrosis model group; Group C, HYSA prevention group; Group D, low dose HYSA group; Group E, moderate dose HYSA group; Group F, high dose HYSA group; Group G, colchicine group. HYSA, hydroxysafflor yellow A; w, weeks.

A

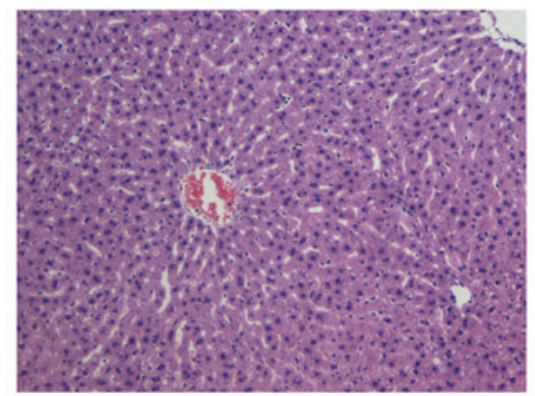

B

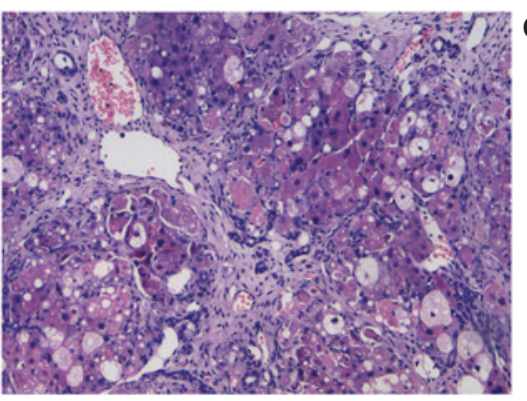

D

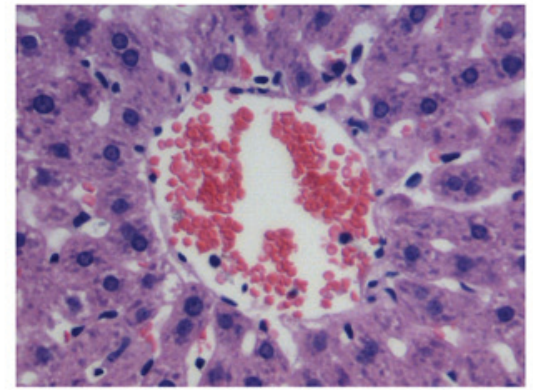

$\mathbf{E}$

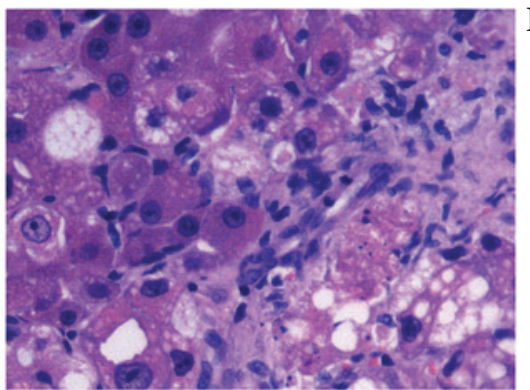

G

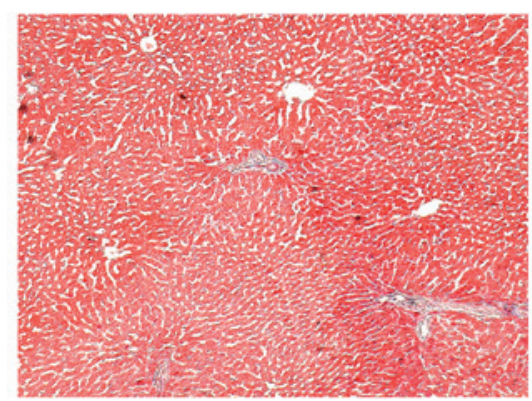

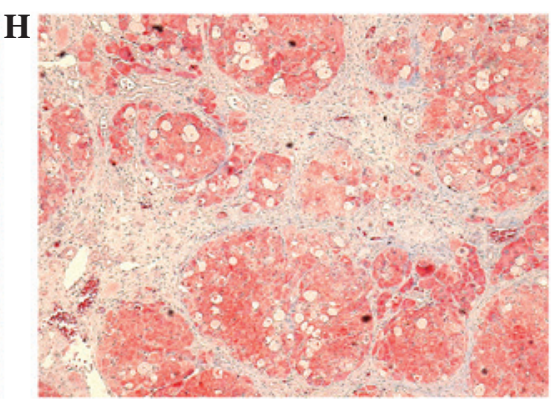
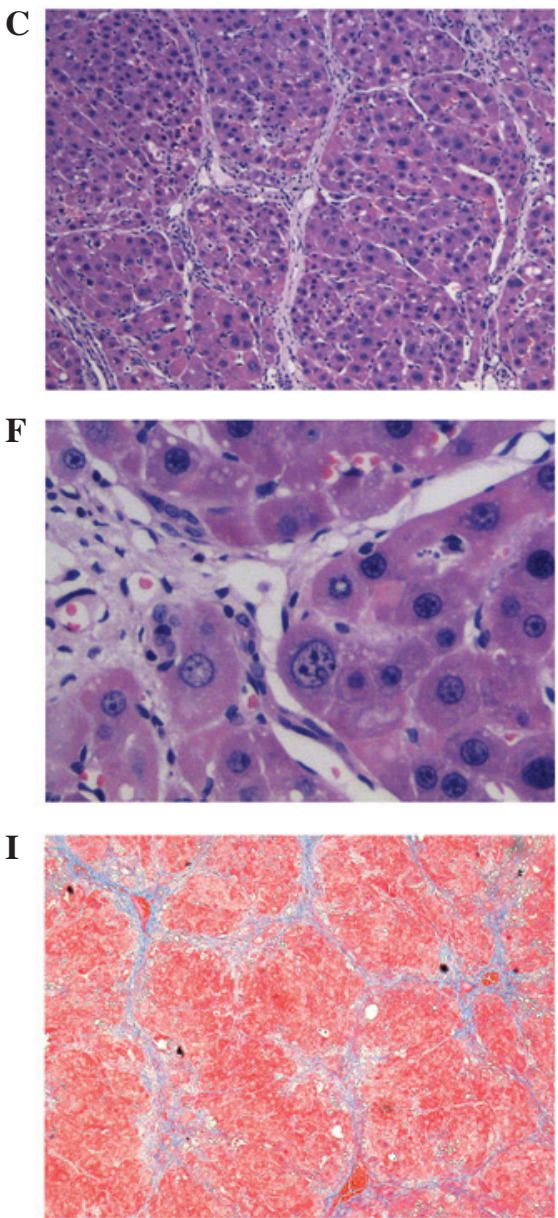

Figure 2. Pathological alterations of liver tissue, as detected by (A-F) hematoxylin-eosin staining and (G-I) Masson staining. (A) Normal liver tissue in the control group. (B) Hepatic lobules of rats in the model group exhibited damage, and developed a typical false flocculus structure. (C) Compared with the model group, hepatic lobules in Group D exhibited reduced structural damage (magnification: x100). (D) Normal liver tissue in the control group. (E) Increased proliferation of collagen fibers, typical pseudolobule formation, hepatic cords, cellular swelling, fatty degeneration, and necrosis and inflammatory cell infiltration were observed in model rats. (F) Compared with the model group, hepatic lobules in Group D exhibited reduced pseudolobule formation, cellular swelling, fatty degeneration, necrosis and inflammatory cell infiltration, particularly in the hydroxysafflor yellow A prevention group (magnification, $\mathrm{x} 400$ ). (G) Normal liver tissue in the control group. $(\mathrm{H})$ Liver tissue of rats in the model group exhibited marked collagen fiber proliferation, predominantly in portal areas and the central vein, and pseudolobule formation was observed. (I) Liver tissue of rats in Group D exhibited decreased deposition of collagen fibers, a thin fibrous septum and decreased pseudolobule formation (magnification: $\mathrm{x} 100$ ). 
A

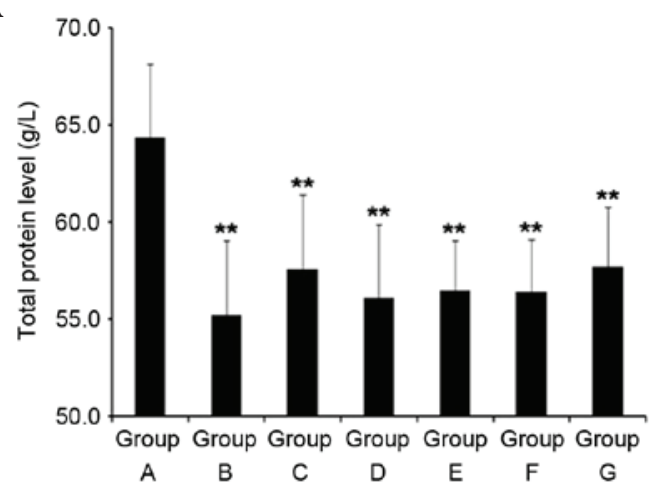

C

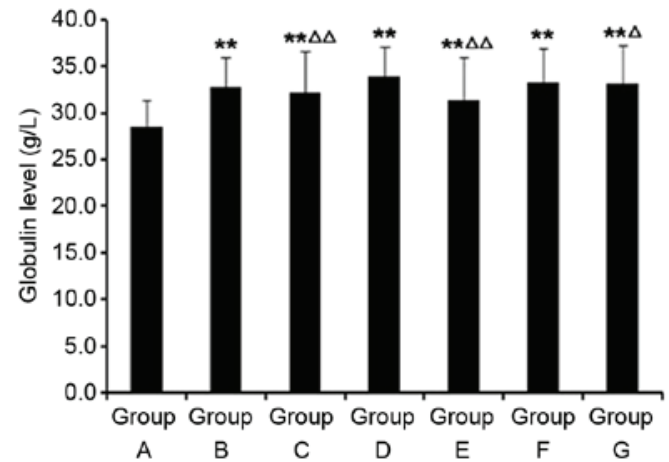

B
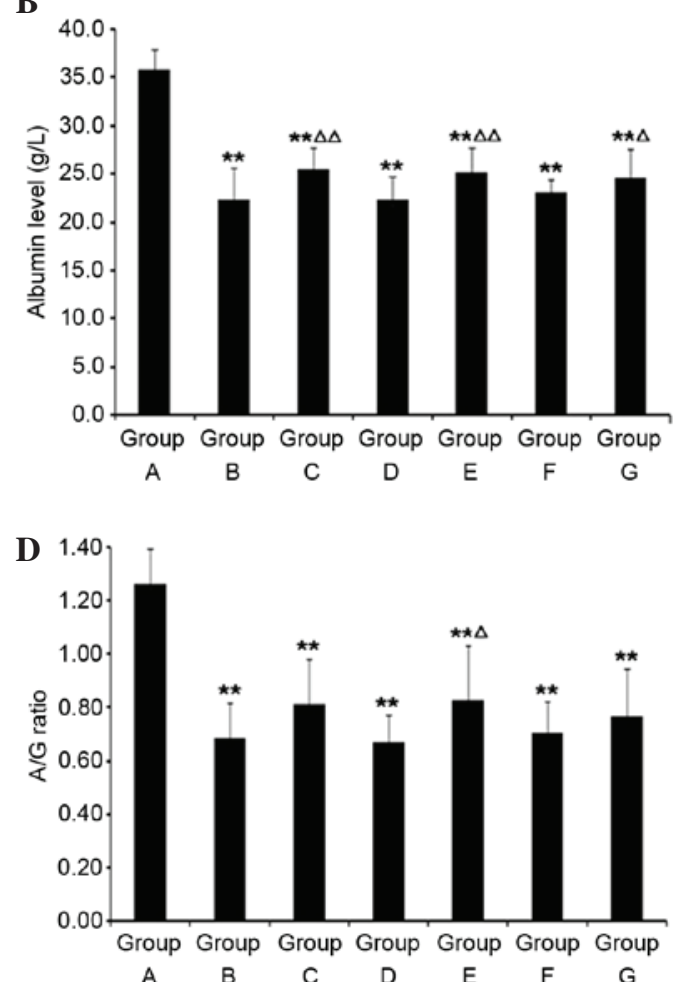

Figure 3. Effects of HSYA on (A) TP, (B) ALB, (C) GLB and (D) A/G ratio in rats with hepatic fibrosis. Compared with in Group A, serum TP, ALB and A/G ratio were significantly decreased in in Group B, and GLB was increased. As compared with Group B, ALB and A/G ratio were increased in drug-treated groups. Serum ALB was particularly increased in Groups $\mathrm{C}$ and $\mathrm{E}$. ${ }^{* * *} \mathrm{P}<0.01$ vs. Group $\mathrm{A} ;{ }^{\triangle \mathrm{P}}<0.05,{ }^{\Delta \wedge} \mathrm{P}<0.01$ vs. Group $\mathrm{B}$. TP, total protein, ALB, albumin; GLB, globulin; A/G, ALB/GLB; Group A, normal control; Group B, hepatic fibrosis model group; Group C, HYSA prevention group; Group D, low dose HYSA group; Group E, moderate dose HYSA group; Group F, high dose HYSA group; Group G, colchicine group. HYSA, hydroxysafflor yellow A.

$\mathbf{A}$

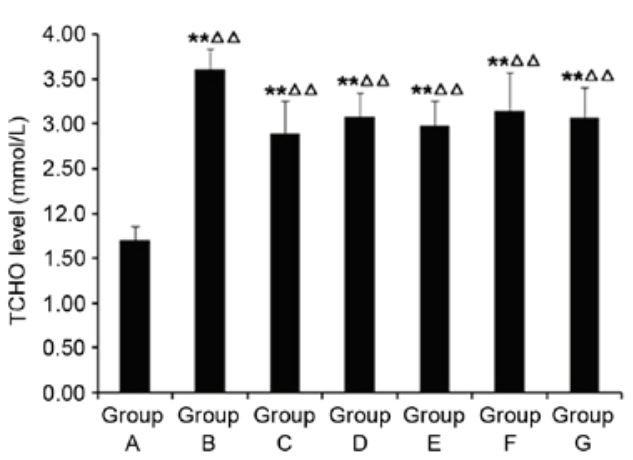

C

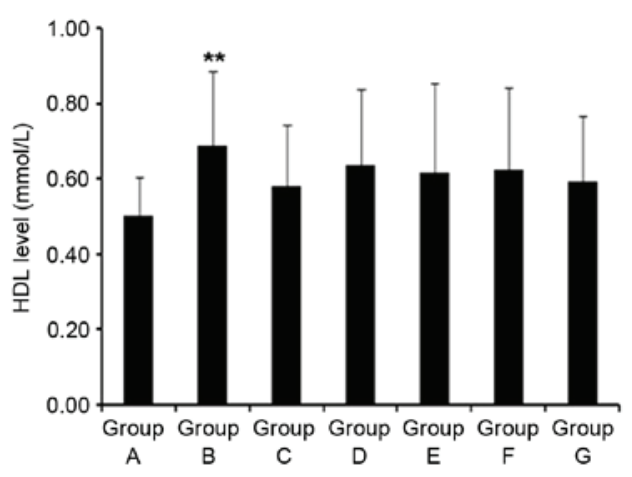

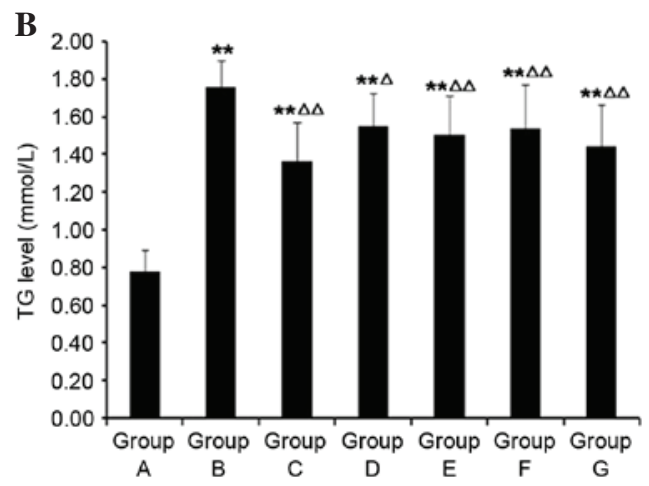

D

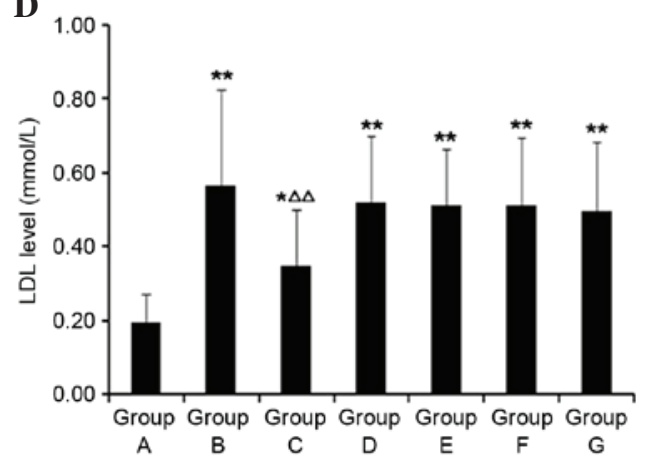

Figure 4. Effects of HSYA on (A) TCHO, (B) TG, (C) HDL and (D) LDL in rats with hepatic fibrosis. Compared with Group A, serum TCHO, TG, HDL and LDL levels were significantly increased in Group B. Compared with in Group B, following HSYA and colchicine treatment, TCHO and TG were decreased, and HDL and LDL had the tendency to decrease. ${ }^{*} \mathrm{P}<0.05,{ }^{* *} \mathrm{P}<0.01$ vs. Group $\mathrm{A} ;{ }^{\Delta} \mathrm{P}<0.05,{ }^{\Delta \Delta} \mathrm{P}<0.01$ vs. Group B. TCHO, total cholesterol; TG, triglycerides; HDL, high-density lipoprotein; LDL, low-density lipoprotein; Group A, normal control; Group B, hepatic fibrosis model group; Group C, HYSA prevention group; Group D, low dose HYSA group; Group E, moderate dose HYSA group; Group F, high dose HYSA group; Group G, colchicine group. HYSA, hydroxysafflor yellow A. 
A

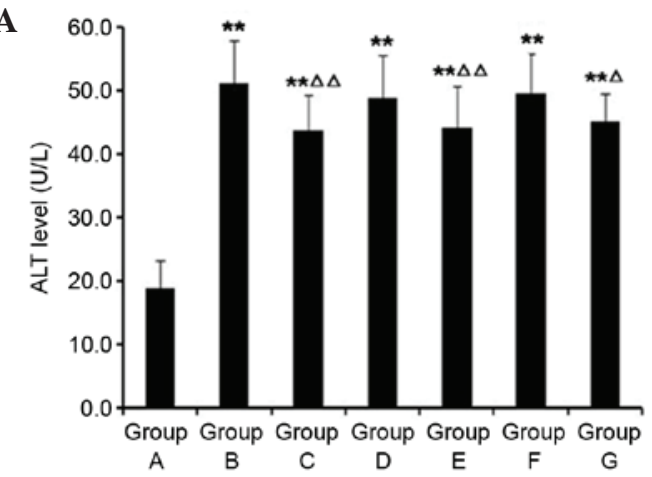

C

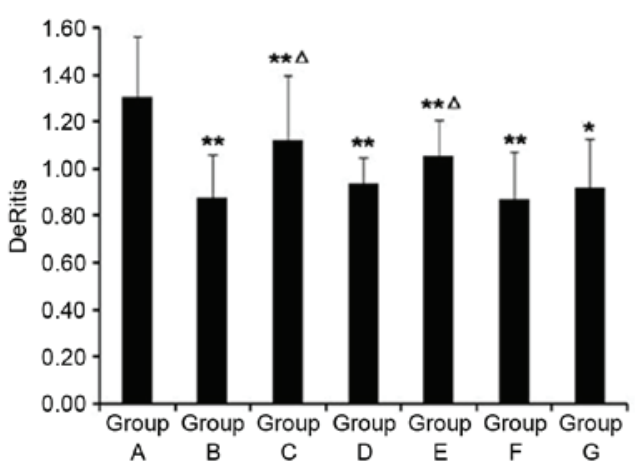

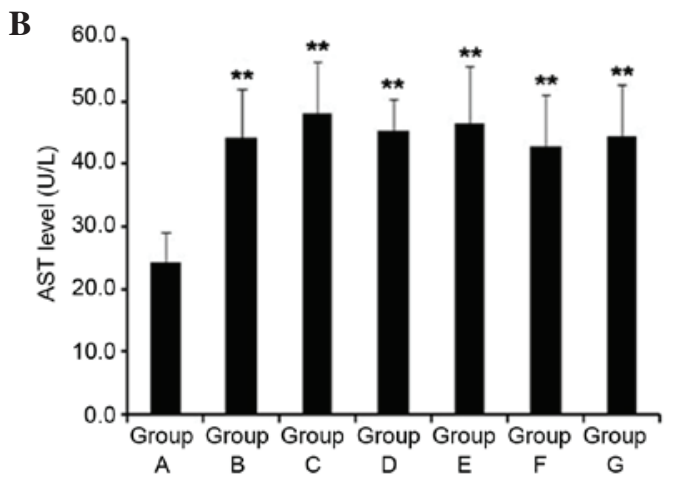

D

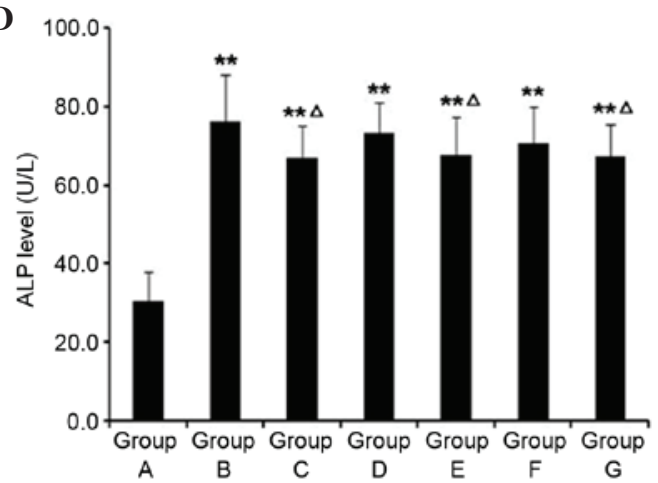

Figure 5. Effects of HSYA on (A) ALT, (B) AST, (C) DeRitis and (D) ALP in rats with hepatic fibrosis. Compared with Group A, ALT, AST and ALP levels were significantly increased in Group B, and the DeRitis value was decreased. Compared with Group B, following treatment with HSYA and colchicine, ALT and ALP levels were decreased, and AST had the tendency to decrease. The DeRitis value was significantly increased in Groups $\mathrm{C}$ and $\mathrm{E}$. ${ }^{*} \mathrm{P}<0.05$, ${ }^{* *} \mathrm{P}<0.01$ vs. Group A; ${ }^{\Delta} \mathrm{P}<0.05,{ }^{\Delta} \mathrm{P}<0.01$ vs. Group B. ALT, alanine aminotransferase; AST, aspartate aminotransferase; ALP, alkaline phosphatase; Group A, normal control; Group B, hepatic fibrosis model group; Group C, HYSA prevention group; Group D, low dose HYSA group; Group E, moderate dose HYSA group; Group F, high dose HYSA group; Group G, colchicine group. HYSA, hydroxysafflor yellow A.

Effects of HSYA on serum hepatic fibrosis index in rats with hepatic fibrosis. Compared with Group A, serum HA, LN, pcIII and cIV levels in Group B were significantly increased $(\mathrm{P}<0.01$; Fig. 6). Compared with Group B, following treatment with HSYA and colchicine, serum HA and LN levels were decreased $(\mathrm{P}<0.05)$. pcIII levels in Group $\mathrm{C}, \mathrm{E}$ and $\mathrm{G}$ were significantly decreased $(\mathrm{P}<0.05)$. Only in Groups $\mathrm{C}$ and $\mathrm{G}$ were serum cIV levels decreased $(\mathrm{P}<0.05)$.

Effects of HSYA on HYP, MDA and SOD in the liver tissue homogenate of rats with hepatic fibrosis. Compared with Group A, HYP and MDA levels in rat liver tissue homogenates from Group B were significantly increased $(\mathrm{P}<0.01$; Fig. 7A and $\mathrm{B}$ ), whereas SOD levels were decreased ( $\mathrm{P}<0.01$; Fig. $7 \mathrm{C}$ ). Compared with Group B, following treatment with HSYA and colchicine, MDA levels in rat liver tissue homogenates were significantly decreased in the treatment groups $(\mathrm{P}<0.05)$, except for Group D. HYP levels in rat liver tissue homogenates were significantly decreased in the treatment groups $(\mathrm{P}<0.05)$. SOD levels were significantly increased in the treatment groups compared with in Group B $(\mathrm{P}<0.05)$, except for Group D.

\section{Discussion}

Hepatic fibrosis, which is commonly seen in hepatic injury and inflammation, and is caused by several pathogenic factors, is a significant process in the development and deterioration of liver cirrhosis and cancer. Hepatic fibrosis is considered an important health concern worldwide, and accounts for $50 \%$ of the mortality rate of patients with liver cancer (16). Despite the high incidence of hepatic fibrosis, no effective therapeutic method is available for clinical treatment. Diversified drugs in Western medicine have been developed for the treatment of hepatic fibrosis, predominantly those used for antiviral therapy and liver protection. These drugs can be effective in treating hepatic fibrosis through inhibiting inflammation, decreasing extracellular matrix proliferation, promoting extracellular matrix degradation, improving microcirculation and decreasing complications. Although the efficacy of these drugs has been demonstrated in clinical research, some problems remain that require resolving $(17,18)$. Traditional Chinese medicine has efficacy for the treatment of complicated diseases and related concerns. The majority of traditional Chinese medicines are used in combination for several targets, including transforming growth factor-SMAD, extracellular signal-related kinase/mitogen-activated protein kinase and the nuclear factor $\kappa \mathrm{B}$ signaling pathway, which can inhibit the progression of hepatic fibrosis and liver cirrhosis via multi-path and multi-level action (19-21).

In Chinese medicine, hepatic fibrosis belongs to the category of hypochondriac pain, accumulation and scar, and the main pathogenesis is considered the deficiency of Qi and blood stasis (22). At present, the majority of studies have focused on promoting circulation and removing stasis (23). Studies regarding the pharmacology of traditional Chinese medicine have demonstrated that promoting circulation and removing 
A

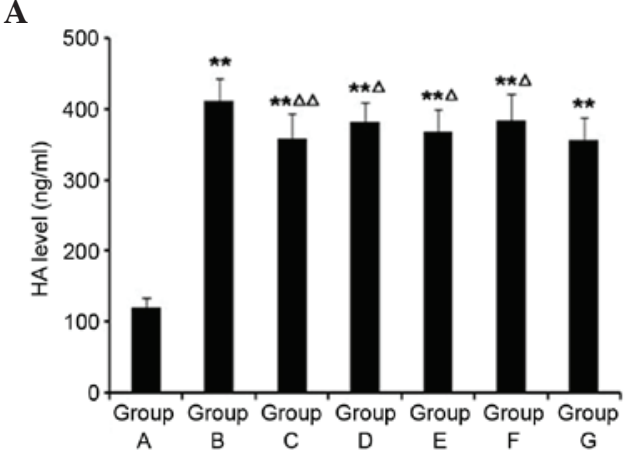

C

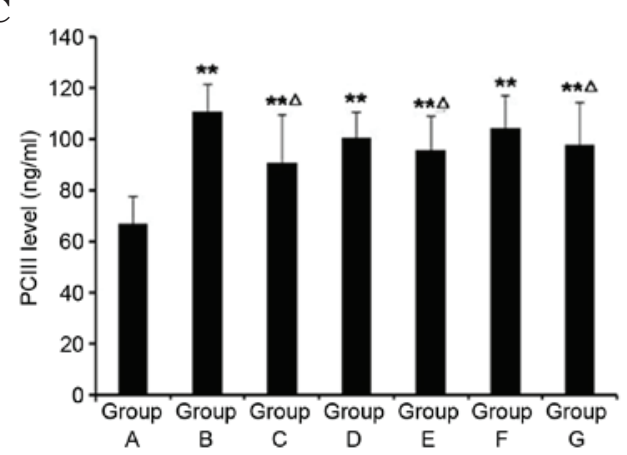

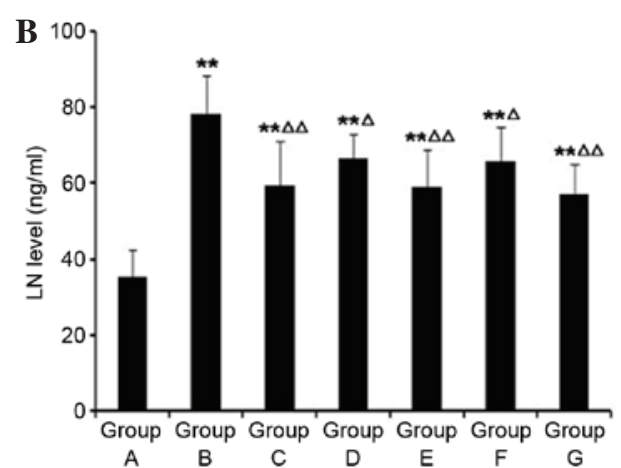

D

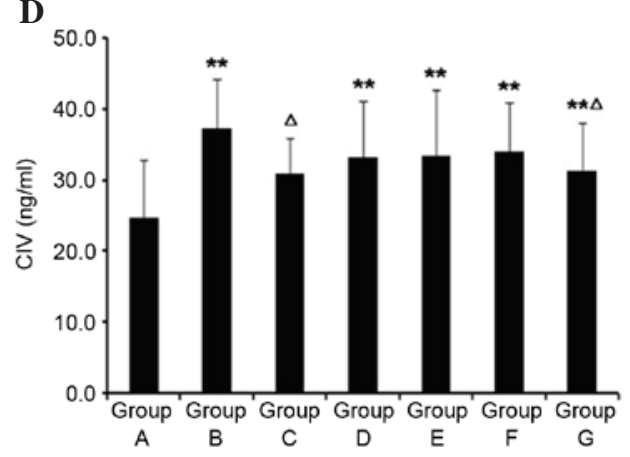

Figure 6. Effects of HSYA on (A) HA, (B) LN, (C) pcIII and (D) cIV in rats with hepatic fibrosis. Compared with Group A, HA, LN, pcIII and cIV levels in Group B were significantly increased. Compared with in Group B, following treatment with HSYA and colchicine, HA and LN levels in each group were decreased. pcIII levels in Groups $\mathrm{C}, \mathrm{E}$ and $\mathrm{G}$ were significantly decreased. Only in Groups $\mathrm{C}$ and $\mathrm{E}$ were serum cIV levels significantly decreased. ${ }^{* *} \mathrm{P}<0.01$ vs. Group A; ${ }^{\Delta} \mathrm{P}<0.05,{ }^{\Delta} \mathrm{P}<0.01$ vs. Group B. HA, hyaluronic acid; LN, laminin; pcIII, procollagen III N-terminal peptide; cIV, collagen type IV; Group A, normal control; Group B, hepatic fibrosis model group; Group C, HYSA prevention group; Group D, low dose HYSA group; Group E, moderate dose HYSA group; Group F, high dose HYSA group; Group G, colchicine group. HYSA, hydroxysafflor yellow A.

stasis results in regulation of hemorheological properties, expands the circumference of blood vessels, increases blood volume of organs and improves microcirculation $(24,25)$. In addition, these drugs can be used to reduce inflammation, induce analgesia and depress hematic fat, enhance the tolerance of cells to hypoxia, increase SOD activity and reduce tissue damage resulting from ischemia-reperfusion injury $(26,27)$. HSYA is a water-soluble monomer extracted from safflower. The present study established a rat model of hepatic fibrosis, and administered HSYA during the process of hepatic fibrosis. Subsequently, the inhibitory effects of HSYA on hepatic fibrosis, and the effects of HSYA on the changes to serum and liver tissue homogenate indices were determined. In particular, the present study explored the mechanism of action of HSYA on hepatic fibrosis.

$\mathrm{CCl}_{4}$-induced hepatic fibrosis is a typical hepatic fibrosis animal model, which is similar to human hepatic fibrosis in morphology and pathophysiology. This model is widely used for studying the pathogenetic mechanism of hepatic fibrosis and for the evaluation of anti-hepatic fibrosis drugs (28). $\mathrm{CCl}_{4}$ is a selective toxicant in the liver, which can produce $-\mathrm{CCl}_{3}$ and $-\mathrm{OOCl}_{3}$ via metabolism of $\mathrm{P} 450$, strengthen lipid peroxidation, destroy the membranous structure of liver cells, and induce liver cell necrosis and disorders in protein synthesis and energy metabolism (29). Furthermore, it can induce activation of hepatic stellate cells and lead to excess deposition of extracellular matrix, finally resulting in hepatic fibrosis. In the present study, rats were simultaneously given ethanol and a high-fat diet. Ethanol can produce toxic metabolites via alcohol dehydrogenase and alcohol oxidase resulting in the loss of liver cells, and can induce P450 activation, accelerate liver cell necrosis and shorten the time of model establishment. High-fat diet can result in increased levels of free fatty acid in the liver. Excess free fatty acid in liver cells can lead to fatty degeneration, activate relevant cytokines, stimulate hepatic stellate cells and result in the development of hepatic fibrosis (30). During the process of the present study, rat mortality increased with extension of the model. However, following treatment with HSYA and colchicine, health of the rats in the treatment groups improved.

The results of the present study demonstrated that HSYA resulted in resistance to $\mathrm{CCl}_{4}$-induced weight loss, and improved the general condition of the rats with hepatic fibrosis. In addition, compared with in Group B, liver samples exhibited improved color and size, and had a smooth surface following treatment, thus suggesting that HSYA intervention during the process of hepatic fibrosis can improve the quality of life of rats. Hematoxylin-eosin and Masson staining demonstrated that HSYA intervention was able to relieve cellular swelling, fatty degeneration, necrosis, inflammatory cell infiltration and fibroblast proliferation, thus suggesting that HSYA could protect against liver damage, and reduce the deposition of collagen and injury of liver cells.

TP, ALB and GLB are important indices that reflect hepatic function, which are predominantly used to detect chronic hepatic injury and reflect the function of storing hepatocytes (31). The present study revealed that TP and ALB levels were significantly decreased in Group B; and the inversion 

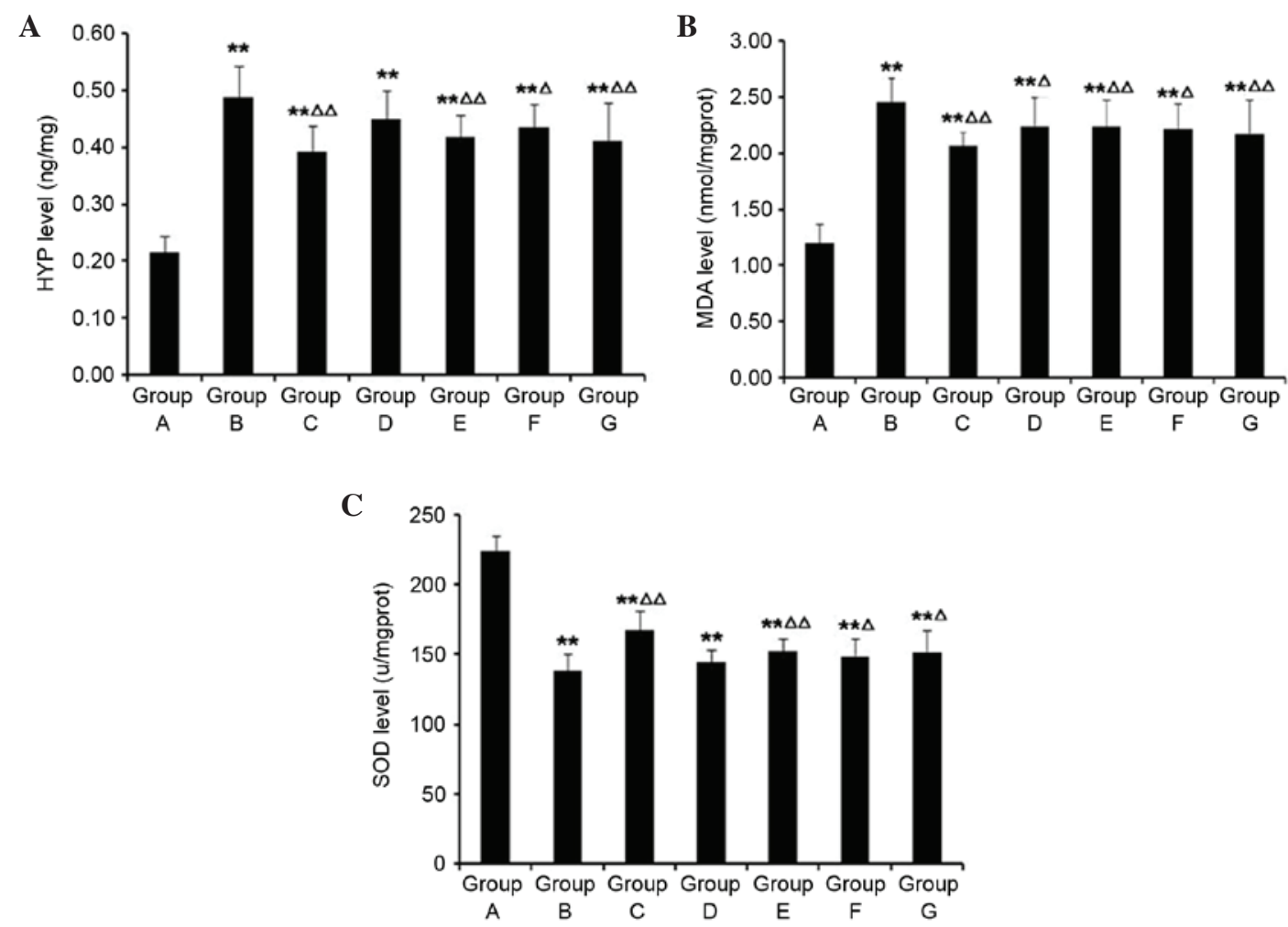

Figure 7. Effects of HSYA on (A) HYP, (B) MDA and (C) SOD levels in rats with hepatic fibrosis. Compared with Group A, HYP and MDA levels were significantly increased in rat liver tissue homogenates in Group B, whereas SOD levels were decreased. Compared with Group B, following treatment with HSYA and colchicine, MDA levels in rat liver tissue homogenates were significantly decreased in each group. HYP levels in rat liver tissue homogenates were significantly decreased in the other groups, except for Group D. SOD levels were significantly increased in the other groups compared with Group B, except for Group D. ${ }^{* *} \mathrm{P}<0.01$ vs. Group A; ${ }^{\Delta} \mathrm{P}<0.05,{ }^{\Delta} \mathrm{P}<0.01$ vs. Group B. HYP, hydroxyproline; MDA, malondialdehyde; SOD, superoxide dismutase; Group A, normal control; Group B, hepatic fibrosis model group; Group C, HYSA prevention group; Group D, low dose HYSA group; Group E, moderate dose HYSA group; Group F, high dose HYSA group; Group G, colchicine group. HYSA, hydroxysafflor yellow A.

of the $\mathrm{A} / \mathrm{G}$ ratio suggested the induction of hepatic function injury. Following treatment with HSYA and colchicine, ALB in Groups $\mathrm{C}$ and $\mathrm{E}$ was increased $(\mathrm{P}<0.01)$, suggesting that ALB content was positively associated with liver cell number and function. Following model establishment, ALB levels were decreased, which indicated severe liver cell necrosis; following HSYA and colchicine treatment, increased ALB levels indicated effective treatment.

Liver cell injury can result in aberrant lipid metabolism, which is predominantly manifested as lecithin cholesterol acyltransferase deficiency and decreased activation of lipoprotein lipase (32). The present study demonstrated that TCHO, TG, HDL and LDL were increased in Group B compared with in Group $A(P<0.01)$, suggesting the presence of aberrant lipid metabolism caused by liver cell injury. Following treatment with HSYA and colchicine, TCHO and TG levels were reduced, suggesting that HSYA may improve lipid metabolism and promote the recovery of liver cell function.

ALT and AST in the cytoplasm release into the plasma when liver cells are damaged and permeability of liver membranes is increased, thus leading to increased ALT and AST activation (33). ALT predominantly exists outside of the mitochondria, whereas $\sim 80 \%$ AST exists in the mitochondria. Moderate liver cell injury leads to an increased leakage of ALT, more so than AST. Severe liver cell injury results in mitochondrial membrane impairment and the release of AST, thus resulting in an increased DeRitis ratio. The majority of
ALP found in the serum is from the liver and skeleton; therefore, serum ALP levels can serve as an examination index for liver diseases (34). The present study demonstrated that serum ALT, AST and ALP levels were increased in Group B $(\mathrm{P}<0.01)$. Higher serum ALT levels, rather than AST levels, and a DeRitis ratio $<1$ indicate a high degree of hepatic fibrosis. Following drug treatment in the present study, the results demonstrated that HSYA was able to reduce the levels of ALT and AST in rats with hepatic fibrosis, particularly in Groups $\mathrm{C}$ and $\mathrm{E}$ $(\mathrm{P}<0.05)$. These results suggested that HSYA can regulate $\mathrm{CCl}_{4}$-induced liver cell injury and improve hepatic function.

Serum HA, LN, pcIII and cIV are degradation products of collagen tissue, which reflects the change of extracellular matrix during the process of liver fibrosis. They are commonly used to diagnose hepatic fibrosis in clinical practice (35). HA is a type of proteoglycan, which is formed due to the covalent binding between protein and glycosaminoglycan. It is synthesized by interstitial cells and is the main component of the extracellular matrix. HA is absorbed into the blood through the lymph, is degraded following uptake by hepatic sinusoidal endothelial cells, and is finally discharged via the liver. The synthesis and discharge of HA is relatively stable, and the serum content of HA is usually very low. HA is significantly synthesized in chronic hepatic disease, particularly in hepatic fibrosis. Due to endothelial cell damage, the uptake and degradation of HA is decreased, resulting in significantly increased levels of serum HA $(36,37)$. 
$\mathrm{LN}$ is a macromolecular noncollagenous glycoprotein that is present in the extracellular matrix of the liver. LN is present in the stratum lucidum of the basement membrane, and the content is usually very low in the serum. LN settles in hepatic sinusoids during fibrosis, and LN content is related to hepatic fibrosis and the degree of inflammatory cell infiltration, promoting hepatic sinusoid capillarization. $\mathrm{LN}$ is a key factor in the induction of portal hypertension (38).

pcIII is an amino-terminal polypeptide formed by collagen III decomposition via amino-terminal peptidase prior to the secretion of collagen III outside the liver for settlement, which can directly reflect the metabolic status of collagen III. During the early stage of hepatic fibrosis, collagen III levels are increased; therefore, pcIII is considered a sensitive indicator for early hepatic fibrosis. Serum pcIII levels are closely associated with the degree of hepatic fibrosis, and its increase is associated with inflammation and necrosis (39).

cIV is combined with LN, and co-exists in the basement membrane of blood vessels, adjusting the adhesion, growth and differentiation of cells. There is no significant cIV settlement in normal hepatic sinusoids; however, during liver fibrosis, cIV synthesis increases, there is a large amount of deposition in the liver blood sinus endothelial cells, alongside LN, which is involved in the formation of liver fibrosis (40). The main pathological alteration associated with hepatic fibrosis is an excessive deposition of several types of extracellular matrix proteins, including collagen, proteoglycan and fibronectin, resulting in hepatic fibrosis, or even cirrhosis. Hepatic fibrosis can be diagnosed by the joint detection of serum HA, LN, pcIII and cIV. Their levels are related to the severity of chronic hepatic disease (41). This study indicated that HA, LN, pcIII and cIV were reduced following treatment with HSYA, which implied an inhibiting effect on synthesis and deposition of rat's extracellular matrix during fibrosis, and then decreased hepatic fibrosis degree. However, further studies regarding the mechanism of action are required.

HYP is a specific amino acid and is a major component of collagenous fiber. HYP content in liver tissue can reflect collagenous fiber content relatively accurately (42). The present study demonstrated that HYP in liver tissue was significantly increased following induction of hepatic fibrosis by $\mathrm{CCl}_{4}$, which indicated active collagen hyperplasia. This result was in accordance with those of previous reports $(43,44)$, thus indicating generation of a successful model. Conversely, HYP content was significantly decreased following treatment with various doses of HSYA. The results from Groups $\mathrm{C}$ and $\mathrm{E}$ were particularly obvious, thus indicating a good preventative effect of HYSA on the degree of hepatic fibrosis in experimental rats.

It has been indicated that single liver cell damage is not enough to induce hepatic fibrosis, however, lipid peroxidation is one of the main links during hepatic fibrosis caused by various factors (45). MDA is the final decomposition product following lipid peroxidation, which is caused by free radicals attacking the polyunsaturated fatty acids in biological membranes; MDA content can be used to reflect the degree of lipid peroxidation in cells (46). SOD is a principal antioxidant, which catalyzes the dismutation of the superoxide anion free radical, resulting in elimination of the superoxide anion free radical and protection of cells from damage (47). In the present study, MDA and SOD levels were detected, indirectly reflecting the degree of lipid peroxidation damage to liver tissue. The results demonstrated that MDA levels in the liver tissue homogenates of Group B rats were significantly increased $(\mathrm{P}<0.01)$, whereas SOD levels were markedly decreased $(\mathrm{P}<0.01)$, indicating severe liver cell damage induced by hepatic fibrosis. Conversely, HSYA could enhance SOD activity and reduce MDA levels, thus inhibiting lipid peroxidation caused by free radicals, inducing protection of liver cell structure and strengthening antioxidant functions. These results indicated that HSYA may be involved in the repair of damaged liver cells.

In conclusion, HSYA serves certain roles in improving hepatic function and alleviating hepatic fibrosis. Its underlying mechanism may be associated with liver cell protection, metabolic adjustment of extracellular matrix molecules, and inhibition of lipid peroxidation. These results provide the experimental basis for the use of HSYA in clinical application for hepatic fibrosis treatment. The cause and pathogenesis of hepatic fibrosis are complex; therefore, it remains unclear the underlying mechanisms of HSYA anti-hepatic fibrosis effects. More comprehensive studies regarding the effects of HYSA at the cell, sub-cell, protein and molecular level are required.

\section{Acknowledgements}

The present study was supported by a grant from the Natural Science Foundation of Shandong Province (grant no. ZR2011HL063), the Natural Science Foundation of Shandong Province (grant no. ZR2011HM073) and the Natural Science Foundation of Shandong Province (grant no. ZR2013HM047). The authors would like to thank Dr. Fenghua Fu (School of Pharmaceutical Sciences, Yantai University, Yantai, China) for providing HSYA, and would like to thank the reviewers for their valuable comments on how to improve the quality of this paper.

\section{References}

1. Zois C, Baltayiannis G, Karayiannis P and Tsianos EV: Systematic review: Hepatic fibrosis-regression with therapy. Aliment Pharmacol Ther 28: 1175-1187, 2008.

2. Bralet MP: Image in pathology. Congenital hepatic fibrosis. Ann Pathol 24: 284, 2004 (In French).

3. Cogliati B, Da Silva TC, Aloia TP, Chaible LM, Real-Lima MA, Sanches DS, Matsuzaki P, Hernandez-Blazquez FJ and Dagli ML: Morphological and molecular pathology of $\mathrm{CCL}_{4}$-induced hepatic fibrosis in connexin43-deficient mice. Microsc Res Tech 74: 421-429, 2011.

4. Wang L, Cheng D, Wang H, Di L, Zhou X, Xu T, Yang X and Liu Y. The hepatoprotective and antifibrotic effects of Saururus chinensis against carbon tetrachloride induced hepatic fibrosis in rats. J Ethnopharmacol 126: 487-491, 2009.

5. Liu P, Hu YY, Liu C, Xu LM, Liu CH, Sun KW, Hu DC, Yin YK, Zhou XQ, Wan MB, et al: Multicentre clinical study On Fuzhenghuayu capsule against liver fibrosis due to chronic hepatitis B. World J Gastroenterol 11: 2892-2899, 2005.

6. Ru QJ, Tang ZM, Zhang ZE and Zhu Q: Clinical observation on effect of xuefu zhuyu decoction in treating patients with liver fibrosis caused by chronic hepatitis B. Chin J Integr Trad Western Med 24: 983-985, 2004.

7. Lu C, Shen Q, Yang J, Wang B and Song C: The complete chloroplast genome sequence of Safflower (Carthamus tinctorius L.). Mitochondrial DNA A DNA Mapp Seq Anal 27: 3351-3353, 2016. 
8. Zhao JF, Liu J, Guo Y, Liu Q, Dai Z, Ma SC and Lin RC: Chemical constituents from safflower injection and their bioactivity. Zhongguo Zhong Yao Za Zhi 39: 3102-3106, 2014 (In Chinese).

9. Li Y, Chen Y, Wang L, Chen X, Liu X, Sun C and Yan W: Research on technological process of two-pot countercurrent extraction of hydroxysafflor yellow A. Zhongguo Zhong Yao Za Zhi 34: 2743-2747, 2009.

10. Bie XD, Han J and Dai HB: Effects of hydroxysafflor yellow A on the experimental traumatic brain injury in rats. J Asian Nat Prod Res 12: 239-247, 2010.

11. Liu SX, Zhang Y, Wang YF, Li XC, Xiang MX, Bian C and Chen P: Upregulation of heme oxygenase-1 expression by hydroxysafflor yellow A conferring protection from anoxia/reoxygenation-induced apoptosis in H9c2 cardiomyocytes. Int J Cardiol 160: 95-101, 2012.

12. Zhu HJ, Wang LJ, Wang XQ, Pan H, Li NS, Yang HB, Jin M, Zang BX and Gong FY: Hormone-sensitive lipase is involved in the action of hydroxysafflor yellow A (HYSA) inhibiting adipogenesis of 3T3-L1cells. Fitoterapia 93: 182-188, 2014.

13. Wang C, Huang Q, Wang C, Zhu X, Duan Y, Yuan S and Bai X: Hydroxysafflor yellow A suppresses oleic acid-induced acute lung injury via protein kinase A. Toxicol Appl Pharmacol 272: 895-904, 2013

14. Chobert MN, Couchie D, Fourcot A, Zafrani ES, Laperche Y, Mavier P and Brouillet A: Liver precursor cells increase hepatic fibrosis induced by chronic carbon tetrachloride intoxication in rats. Lab Invest 92: 135-150, 2012.

15. Wang XH, Lu XF, Liu CY, Hu SJ, Kang XX and Yang J (eds): Diagnostics. 8th edition. People's Health Publishing House, p352, 2015 (In Chinese).

16. Friedman SL: Hepatic fibrosis-overview. Toxicology 254: 120-129, 2008

17. Guo J and Friedman SL: Hepatic fibmgenesis. Semin Liver Dis 27: 413-426, 2007.

18. Spengler U: Hepatic microcirculation: A critical but neglected factor for the outcome of viral hepatitis. J Hcpatol 50: 631-633, 2009.

19. Liu C, Wang G, Chen G, Mu Y, Zhang L, Hu X, Sun M, Liu C and Liu P: Huangqi decoction inhibils apoptosis and fibrosis, but promotes Kupffer cell activation in dimefhyllnitrosamine-induced rat liver fibrosis. BMC Complement Altern Med 12: 51, 2012.

20. Wang Q, Wen R, Lin Q, Wang N, Lu P and Zhu X: Wogonoside shows antifibrotic effects in an experimental regression model of hepatic fibrosis. Dig Dis Sci 60: 3329-3339, 2015.

21. Yang F, Li J, Zhu J, Wang D, Chen S and Bai X: Hydroxysafflor yellow A inhibits angiogenesis of hepatocellular carcinoma via blocking ERK/MAPK and NF- $\mathrm{BB}$ signaling pathway in $\mathrm{H} 22$ tumor-bearing mice. Eur J Pharmacol 754: 105-114, 2015.

22. Liu C, Wang G, Chen G, Mu Y, Zhang L, Hu X, Sun M, Liu C and Liu P: Huangqi decoction inhibits apoptosis and fibrosis, but promotes Kupffer cell activation in dimethylnitrosamine-induced rat liver fibrosis. BMC Complement Altern Med 12: 51, 2012

23. Yang XH, Wang DD and Zhu Y: Recent research progress on hydroxysafflor yellow A. J Trad Chin Med Univ Hunan 33: 102-106, 2013 (In Chinese)

24. Liu SY, Zhang YQ, Liu YL, Guo P and Zhou CM: Intervention of chronic hepatitis B liver fibrosis patients in different stages by syndrome typing and different activating blood removing stasis methods: A clinical study. Zhongguo Zhong Xi Yi Jie He Za Zhi 33: 1457-1461, 2013 (In Chinese)

25. Shi XF, Xu M and Liu Q: The effects of total saponins of panax notoginseng on I, III-type collage and TGF in liver of cirrhosis rats. Zhongyao Yaoli Yu Linchuang 17: 7-8, 2001.

26. Wynn TA: Cellular and molecular mechanisms on fibrosis. J Pathl 214: 199-210, 2008

27. Friedman SL, Rockey DC and Bissell DM: Hepatic fibrosis 2006: Report of the third SSALD single topic conference. Hepatology 45: 242-249, 2007.

28. Krählenbühl S, Reichen J, Zimmermann A, Gehr P and Stucki J: Mitochondrial structure and function in $\mathrm{CCl}_{4}$-induced cirrhosis in the rat. Hepatology 12: 526-532, 1990.

29. Domitrović R, Rashed K, Cvijanović O3, Vladimir-Knežević S, Škoda M and Višnić A: Myricitrin exhibits antioxidant, anti-inflammatory and antifibrotic activity in carbon tetrachloride-intoxicated mice. Chem Biol Interact 230: 21-29, 2015.
30. Wang ZB, Huang ZM, Wang JJ, Wu JM, Chen XR, Wu JS and Zhang QY: Inducing rat liver cirrhosis by adjusting the dosage of $\mathrm{CCl}_{4}$ according to body weight changes. Zhonghua Gan Zang Bing Za Zhi 16: 234-235, 2008 (In Chinese).

31. Gonzalez M, Sealls W, Jesch ED, Brosnan MJ, Ladunga I, Ding X, Black PN and DiRusso CC: Defining a relationship between dietary fatty acids and the cytochrome P450 system in a mouse model of fatty liver disease. Physiol Genomics 43: 121-135, 2011.

32. Wang CC, Lim LY, Deubner H, Tapia K, Lau AW, Manansala J, Krows M, Shuhart MC and Kowdley KV: Factors predictive of significant hepatic fibrosis in adults with chronic hepatitis B and normal serum ALT. J Clin Gastroenterol 42: 820-826, 2008.

33. Park JH, Park CK, Kim ES, Park SY, Jo CM, Tak WY, Kweon YO, Kim SK and Choi YW: The diagnostic value of serum hyaluronic acid, 7S domain of type IV collagen and AST/ALT ratio as markers of hepatic fibrosis in chronic hepatitis B and cirrhosis patients. Taehan Kan Hakhoe Chi 9: 79-88, 2003.

34. Li CH, Piao DM, Xu WX, Yin ZR, Jin JS and Shen ZS: Morphological and serum hyaluronic acid, laminin and type IV collagen changes in dimethylnitrosamine-induced hepatic fibrosis of rats. World J Gastroenterol 11: 7620-7624, 2005.

35. Khan JA, Khan FA, Dilawar M, Ijaz A, Khan NA and Mehmood T: Serum hyaluronic acid as a marker of hepatic fibrosis. J Coll Physicians Surg Pak 17: 323-326, 2007.

36. Geramizadeh B, Janfeshan K and Saberfiroozi M: Serum hyaluronic acid as a noninvasive marker of hepatic fibrosis in chronic hepatitis B. Saudi J Gastroenterol 14: 174-177, 2008.

37. Huang Z, Li Q and Wang Z: Observation on dynamic changes of serum procollagen III, hyaluronic acid and laminin in rats with hepatic fibrosis treated with Hujin pill. Zhongguo Zhong Xi Yi Jie He Za Zhi 20: 447-449, 2000 (In Chinese).

38. Tsutsumi M, Takase S, Urashima S, Ueshima Y, Kawahara $\mathrm{H}$ and Takada A: Serum markers for hepatic fibrosis in alcoholic liver disease: Which is the best marker, type III procollagen, type IV collagen, laminin, tissue inhibitor of metalloproteinase, or prolyl hydroxylase? Alcohol Clin Exp Res 20: 1512-1517, 1996.

39. Iushchuk ND, Znoı̌ko OO, Safiullina NKh, Dudina KR, Kelli EI, Klimova EA, Kashirin VI, Braginskiǔ DM, Kushlinskiı̌ NE, Liubimova NV, et al: Diagnostic significance of type IV collagen and hyaluronic acid in the serum of patients with chronic hepatitis C for staging hepatic fibrosis. Ter Arkh 77: 50-55, 2005 (In Russian).

40. Toyoki Y, Sasaki M, Narumi S, Yoshihara S, Morita T and Konn M: Semiquantitative evaluation of hepatic fibrosis by measuring tissue hydroxyproline. Hepatogastroenterology 45: 2261-2264, 1998.

41. Liu J, Tan H, Sun Y, Zhou S, Cao J and Wang F: The preventive effects of heparin-superoxide dismutase on carbon tetrachloride-induced acute liver failure and hepatic fibrosis in mice. Mol Cell Biochem 327: 219-228, 2009.

42. Huang Q, Li Y, Zhang S, Huang R, Zheng L, Wei L, He M, Liao M, Li L, Zhuo L and Lin X: Effect and mechanism of methyl helicterate isolated from Helicteres angustifolia (Sterculiaceae) on hepatic fibrosis induced by carbon tetrachloride in rats. J Ethnopharmacol 143: 889-895, 2012.

43. Kanno K, Tazuma S and Chayama K: AT1A-deficient mice show less severe progression of liver fibrosis induced by $\mathrm{CCl}(4)$. Biochem Biophys Res Commun 308: 177-183, 2003.

44. Smyth R, Munday MR, York MJ, Clarke CJ, Dare T and Turton JA: Comprehensive characterization of serum clinical chemistry parameters and the identification of urinary superoxide dismutase in a carbon tetrachloride-induced model of hepatic fibrosis in the female Hanover Wistar rat. Int J Exp Pathol 88: 361-376, 2007.

45. Yu J, Wang Y, Qian H, Zhao Y, Liu B and Fu C: Polyprenols from Taxus chinensis var. mairei prevent the development of $\mathrm{CCl}_{4}$-induced liver fibrosis in rats. J Ethnopharmacol 142: 151-160, 2012.

46. Liu J, Tan H, Sun Y, Zhou S, Cao J and Wang F: The preventive effects of heparin-superoxide dismutase on carbon tetrachloride-induced acute liver failure and hepatic fibrosis in mice. Mol Cell Biochem 327: 219-228, 2009.

47. Ljubuncic P, Abu-Salach O and Bomzon A: Ursodeoxycholic acid and superoxide anion. World J Gastroenterol 11: 4875-4878, 2005. 